\title{
Control of bovine respiratory disease, with and without co-morbidity by otitis media, in dairy heifers comparing gamithromycin, tulathromycin, or no medication at a commercial development facility
}

\author{
Richard D. Linhart ${ }^{1 *}$ and Gordon W. Brumbaugh ${ }^{2}$ \\ ${ }^{1}$ Boehringer Ingelheim Animal Health, 3239 Satellite Blvd., Duluth, GA 30096 \\ ${ }^{2}$ Private contractor, New Plymouth, ID 83655
}

\section{ABSTRACT}

The objective of this study was to evaluate one strategy for control (metaphylaxis) of bovine respiratory disease, with and without co-morbidity with otitis media, in dairy heifers at a commercial development facility. Individual heifers were the experimental unit. At weaning, 1 of 3 experimental treatments (gamithromycin, tulathromycin, or no medication) was randomly assigned to 1,567 heifers from 11 different dairies. Gamithromycin was administered to 631 heifers, tulathromycin was administered to 621 heifers, and no medication was administered to 315 heifers (negative control). Heifers were then commingled and penned according to body weight. Each pen contained heifers from each group, and periodically, larger numbers of heifers were penned together. All heifers were observed for the subsequent $42 \mathrm{~d}$ and treated according to protocols prescribed for the facility. Morbidity due to respiratory disease was less for heifers medicated with gamithromycin than for heifers medicated with tulathromycin. Morbidity due to respiratory disease was less for heifers medicated with gamithromycin than for heifers in the negative control group. Fewer heifers medicated with either antimicrobial were subsequently treated because of co-morbidity with otitis media. Mortality was not different among the treatment groups. Heifers medicated with either antimicrobial had greater average daily gain than did heifers in the negative control group.

Key words: dairy heifer, respiratory disease metaphylaxis, gamithromycin, tulathromycin

\section{INTRODUCTION}

Optimal development of a dairy heifer requires attention to maintaining its health and well-being from conception through culling from the lactating herd

Received October 8, 2018.

Accepted February 1, 2019.

*Corresponding author: richard.linhart@boehringer-ingelheim.com
(Wells et al., 1997). Bach (2011), Stanton et al. (2012), Guterbock (2014), and Schaffer et al. (2016) are among those that have reported the negative effects of disease (particularly respiratory disease) on eating behavior, growth, and future productivity of the heifer. Respiratory disease was the most common condition affecting weaned dairy heifers and occurred in $11.2 \%$ (SEM = $2.5 \%$ ) of weanling dairy heifers (USDA-APHIS-VSCEAH-NAHMS, 2012). Of those, $97.9 \%($ SEM $=1.7 \%)$ were treated with antimicrobial medication. Antimicrobials most commonly used were florfenicol, a macrolide, or a fluoroquinolone.

Factors associated with the risk of bovine respiratory disease (BRD) in dairy heifers through weaning include dystocia, failure of passive transfer, low birth weight, and stressors such as adverse weather, weaning, commingling in group housing, and co-morbidity (e.g., diarrhea, otitis media, navel infection, and lameness; Bach, 2011; Stanton et al., 2012; USDA-APHIS-VSCEAH-NAHMS, 2012; Walker et al., 2012; Guterbock, 2014; Windeyer et al., 2014; Schaffer et al., 2016). Management practices that help reduce risks of calfhood diseases include cleanliness/hygiene; sufficient and well-trained personnel; appropriate and safe equipment; attended parturition; assurance that the calf receives adequate, high-quality colostrum; proper and adequate nutrition; protection from adverse weather (hypothermia as well as hyperthermia); comfortable bedding; adequate space; and appropriate use of biologicals and antimicrobials.

Bovine respiratory disease and co-morbidities of dairy heifers, may occur from early life through adulthood (USDA-APHIS-VS-CEAH-NAHMS, 2012; Windeyer et al., 2014). The dairy heifer appears to live with the risk of developing BRD at any time during her life. The risk of developing BRD increases when the heifer is moved from individual housing (hutch) to group housing. After removal from the hutch, initial commingling in small groups (approximately 10 head) results in lower incidence of BRD than does initial commingling in larger groups (approximately 25 head). In addition 
to appropriate use of biological products to prevent specific diseases, antimicrobial medications may be administered to cattle at times of predictable high risk for developing BRD or to control BRD. Factors associated with high risk of the development of BRD are essentially the same for dairy heifers as for beef cattle and include dystocia, immune status of the dam, adequate passive transfer, co-morbidity, inherent immune function, hygiene, commingling, transport-related stress, and parturition-related stress (BRD in adults; Fulton, 2009; Gorden and Plummer, 2010; Taylor et al., 2010; Guterbock, 2014).

The objective of this clinical study was to compare the control of BRD in dairy heifers using gamithromycin (Zactran, Merial Inc., Duluth, GA; now part of Boehringer Ingelheim Vetmedica Inc., Duluth, GA), tulathromycin (Draxxin, Zoetis, Parsippany, NJ), or no medication administered at weaning. The null hypothesis $\left(\mathrm{H}_{0}\right)$ was that no detectable difference in morbidity due to BRD would be measured for heifers in the 3 experimental groups. The alternative hypothesis $\left(\mathrm{H}_{1}\right)$ was that different morbidity due to BRD would be measured for heifers in the 3 experimental groups.

\section{MATERIALS AND METHODS}

\section{Facility}

This comparative clinical study was performed at a commercial calf-ranch in the western United States that received calves (primarily heifers) from multiple dairies and developed those heifers until about 6 mo of age. The facility had a one-time capacity of approximately 10,000 head. Extensive records allowed data about the heifers to be retrieved, evaluated, and used to drive management practices (health care, nutrition, grouppenning, and personnel). Historically, when the heifers were weaned, moved from individual housing (hutches), and commingled in group pens, they experienced an increased incidence of BRD during the subsequent several days. The calf-ranch employed an experienced nutritionist and an attending veterinarian. Written protocols were provided for personnel who routinely monitored the health and well-being of the heifers. Personnel (approximately 300 head of weaned heifers/ person) were trained and actively participated in making improvements at the facility. Based on the timing of previous outbreaks of $\mathrm{BRD}$, it was determined that an antimicrobial would be administered when heifers had a high risk of developing BRD during transfer from individual hutches to group housing. Two commercially available macrolide antimicrobial products were compared with each other and with no medication.

\section{Animals}

Dairy-breed (Holstein, Jersey, and Holstein-Jersey cross) heifers arrived at the calf-ranch at $1 \mathrm{~d}$ of age, were weighed, and were placed in sanitized individual hutches. Colostral, passive transfer of antibodies was nonspecifically assessed by determining the concentration of total protein in serum (by digital refractometer) from each heifer at $2 \mathrm{~d}$ of age. Heifers were fed, monitored for health problems, treated, and vaccinated according to written protocols until they were approximately $80 \mathrm{~d}$ of age. The heifers were then weaned, removed from individual hutches, weighed, and housed in commingled groups of 12 head for 3 wk; then, in groups of 24 head for $1 \mathrm{wk}$; then, in groups of 48 head for $1 \mathrm{wk}$; then, in groups of 96 for 1 wk. Heifers were then moved to a large-pen feedlot until they were approximately 6 mo of age. At that time, they were taken to another custom facility or returned to the respective dairy of origin for further development, to be bred, to freshen, and to enter the milking herd.

\section{Experimental Design}

A randomized complete block design was initially considered with pen as the block; however, the penning procedures during the conduct of the study made it necessary to use a completely randomized design. Individual heifers were identified with a unique number and were the experimental units. Experimental treatments were randomly assigned and administered as described below. After administration of the assigned test article, the heifers were commingled (as described above) throughout the subsequent 42-d observation period. Enrollment occurred from October 24, 2016, through November 10, 2016, and heifers enrolled on different days were not commingled during the first 3 wk. The dates of enrollment, the 3 breeds, and the 11 dairies of origin were variables that were randomized among the 3 experimental treatments. Incidence of morbidity due to BRD during the $42-\mathrm{d}$ observation period was the primary variable of interest. Other variables included success of the first treatment because of BRD, BRD case fatality rate, overall mortality, and average daily gain. Feed consumption, feed efficiency, feeding activity, and metabolic activity were not measured. The study was designed with the assumption of $10 \%$ morbidity due to BRD in the heifers that were not medicated (negative control group), $3 \%$ morbidity in the heifers that were medicated with gamithromycin, and $3 \%$ morbidity in the heifers that were medicated with tulathromycin. Power $[1-\beta$ (type II error)] calculations revealed that the targeted number of animals required to detect that 
difference with type I error $(\alpha)=0.05$ was negative control, 300 head; gamithromycin, 600 head; and tulathromycin, 600 head, for a total of 1,500 head.

\section{Procedures}

All activities involved with care and disposal of animals were performed in compliance with applicable federal, state, and local laws and regulations. At the time of weaning (approximately $80 \mathrm{~d}$ of age), heifers were removed from individual hutches and individually weighed, and the test article was randomly assigned and administered. Confirmation and recording of the assigned experimental treatment was made by at least 2 individuals. Negative control was defined as no injection. Test articles were administered subcutaneously according to instructions on the respective label: gamithromycin, $6 \mathrm{mg} / \mathrm{kg}$ of $\mathrm{BW}$, and tulathromycin, $2.5 \mathrm{mg} / \mathrm{kg}$ of BW. Based on previous data, the heifers were expected to weigh approximately $95.5 \mathrm{~kg}$. To meet the protocol for the facility, the respective volumes for the commercial products that contained the experimental treatments were gamithromycin, $4 \mathrm{~mL}$, and tulathromycin, $2.5 \mathrm{~mL}$. The notable difference of dose-volume precluded blinding of the randomly assigned experimental treatment. Separate multi-dose, manually filled, pistol-grip syringes (Allflex USA Inc., DFW Airport, TX) were used to administer the respective medication. Needles $(16$ ga. $\times 1.56 \mathrm{~cm})$ were changed after 10 injections, or when otherwise indicated, according to guidelines (Beef Quality Assurance, 2013). Heifers were then commingled and penned (12 head/pen) according to weight $(<90.9 \mathrm{~kg}$ or $\geq 90.9 \mathrm{~kg})$ in compliance with routine management procedures. Each pen contained heifers of each experimental group and heifers from different dairies of origin.

Following enrollment, heifers were fed twice daily (approximately 0700 and $1500 \mathrm{~h}$ ). Heifers in groups of 12 head were observed by trained personnel twice daily, and those in groups of $\geq 24$ head were observed once daily. Personnel recorded data and continued other routine management practices according to protocols of the calf-ranch. No heifer (including those in the negative control group) was allowed to be treated because of BRD for $2 \mathrm{~d}$ (enforced, $2 \mathrm{~d}$, post-metaphylactic interval). After that enforced interval, heifers with BRD score $>4$ (University of California-Davis, 2016; Table 1) were treated according to the following written protocol: first treatment (6 mg of gamithromycin/ $\mathrm{kg}$ of BW, s.c.), followed by a 7-d interval in which no other medication was administered; second treatment (Resflor Gold; Intervet/Merck Animal Health, Madison, NJ; $40 \mathrm{mg}$ of florfenicol $+2.2 \mathrm{mg}$ of flunixin
Table 1. Scoring system used in this study to record clinical signs post-metaphylaxis (University of California, Davis) ${ }^{1}$

\begin{tabular}{|c|c|c|}
\hline Clinical sign ${ }^{2}$ & $\begin{array}{l}\text { Score if } \\
\text { normal }\end{array}$ & $\begin{array}{c}\text { Score if } \\
\text { abnormal }\end{array}$ \\
\hline Ocular discharge & 0 & 2 \\
\hline Nasal discharge & 0 & 4 \\
\hline Drooping ear(s), head tilt, or both & 0 & 5 \\
\hline Cough & 0 & 2 \\
\hline Breathing & 0 & 2 \\
\hline Rectal temperature & 0 & 2 \\
\hline
\end{tabular}

${ }^{1}$ When the sum of the scores for clinical signs was $>4$, the heifer was treated according to the written protocol.

${ }^{2}$ If drooping ears or head tilt (or both) was the only clinical sign, the condition was classified as otitis media. If cough, abnormal breathing, and abnormal rectal temperature were also present, the condition was classified as bovine respiratory disease (BRD) and otitis media. If clinical signs were present without drooping ears or head tilt (or both), the condition was classified as BRD only.

meglumine/kg of BW, s.c.), followed by a 3 -d interval in which no other medication was administered; and third treatment $(2.5 \mathrm{mg}$ of tulathromycin $/ \mathrm{kg}$ of $\mathrm{BW}$, s.c.), followed by a 7 -d interval in which no other medication was administered.

On d 42 following enrollment, the study was completed, heifers were individually weighed, and data were summarized. All pre-slaughter withdrawal times were observed. A gross necropsy was performed on heifers that died during the study to determine the cause of death. Disposal of the remains was according to applicable regulations. After enrollment, the first treatment because of BRD (BRD morbidity $=\mathrm{BRD}$ only + BRD and otitis media; Tables 2 and 3) was reflective of failure to control BRD. Data for heifers that died were removed ("deads-out") from analyses of ADG. Treatment success (BRD treatment success) was defined as "treated because of BRD, with or without otitis media, only once and did not die during the study." Case fatality rate (BRD case fatality rate) was defined as "treated because of BRD, with or without otitis media, and died because of BRD." Heifers treated because of otitis media (treated: otitis media) was defined as BRD and otitis + otitis only (Table 3). Assessment was made of the risk (risk reduction, relative risk reduction, attributable/actual risk reduction, and number needed to treat) for developing $\mathrm{BRD}$, with or without otitis media (risk of failure to control BRD) after receiving 1 of the 3 experimental treatments, during the subsequent observation period of $42 \mathrm{~d}$ (Straus et al., 2005).

\section{Statistical Analysis}

During analysis, experimental groups were coded to maintain blinding. General and generalized linear mod- 
Table 2. Heifers classified with the clinical condition of bovine respiratory disease (BRD) morbidity (post-metaphylaxis) used for this study $^{1}$

\begin{tabular}{lccc}
\hline Treatment group & $\begin{array}{c}\text { BRD } \\
\text { morbidity }^{2}\end{array}$ & $\begin{array}{c}\text { BRD } \\
\text { only }^{3}\end{array}$ & $\begin{array}{c}\text { BRD } \\
\text { and otitis }\end{array}$ \\
\hline Control & $151 / 315$ & $143 / 315$ & $8 / 315$ \\
Tulathromycin & $(47.94)^{\mathrm{a}}$ & $(45.40)$ & $(2.54)$ \\
& $257 / 621$ & $240 / 621$ & $17 / 621$ \\
Gamithromycin & $(41.38)^{\mathrm{a}}$ & $(38.65)$ & $(2.74)$ \\
& $218 / 631$ & $209 / 631$ & $9 / 631$ \\
& $(34.55)^{\mathrm{b}}$ & $(33.12)$ & $(1.43)$ \\
\hline
\end{tabular}

${ }^{\mathrm{a}, \mathrm{b}}$ Values in the same column with different superscript are significantly different (Table 6).

${ }^{1}$ The clinical condition was used to determine treatment (see Table 1) and for statistical analyses (see Table 6). Values are the number of heifers with the clinical condition/number enrolled (\% in parentheses) by treatment group.

${ }^{2}$ Heifers classified as "BRD morbidity" for statistical analysis (see Table 6) were those classified as "BRD only" and "BRD and otitis."

${ }^{3}$ Heifers with clinical signs of BRD without signs of otitis media.

${ }^{4}$ Heifers with clinical signs of BRD and clinical signs of otitis media.

els were used for all analyses. Models were fitted using binomial (e.g., health) or normal (e.g., ADG) distributions, maximum likelihood estimation, complementarylog-log link, Kenward-Roger degrees of freedom and Newton-Raphson and Ridging optimization procedures (Proc GLIMMIX SAS 9.3, SAS Institute Inc., Cary, NC). Fixed effects included the experimental treatment structure as described above. Model-adjusted means and standard errors of the means were reported for all outcomes (back-transformed to the original scale for generalized models). When overall treatment effects were significant at $P \leq 0.05$ (i.e., reject the null hypothesis that no difference was present among the groups), pairwise comparisons among the groups were performed, again utilizing $P \leq 0.05$ for significance testing (with Tukey adjustment for multiple comparisons).
Table 3. Heifers classified with the clinical condition of treated: otitis media (post-metaphylaxis) used for this study ${ }^{1}$

\begin{tabular}{lccc}
\hline $\begin{array}{l}\text { Treatment } \\
\text { group }\end{array}$ & $\begin{array}{c}\text { Treated: } \\
\text { otitis media }\end{array}$ & $\begin{array}{c}\text { BRD } \\
\text { and otitis }\end{array}$ & $\begin{array}{c}\text { Otitis } \\
\text { only }^{4}\end{array}$ \\
\hline Control & $51 / 315$ & $8 / 315$ & $43 / 315$ \\
Tulathromycin & $(16.19)$ & $(2.54)$ & $(13.65)$ \\
& $77 / 621$ & $17 / 621$ & $60 / 621$ \\
Gamithromycin & $(12.40)$ & $(2.74)$ & $(9.66)$ \\
& $81 / 631$ & $9 / 631$ & $72 / 631$ \\
& $(12.84)$ & $(1.43)$ & $(11.41)$ \\
\hline
\end{tabular}

${ }^{1}$ The clinical condition was used to determine treatment (see Table 1) and for statistical analyses (see Table 6). Values are the number of heifers with the clinical condition/number enrolled (\% in parentheses) by treatment group.

${ }^{2}$ Heifers classified as "treated: otitis media" for statistical analysis (see Table 6) were those classified as "BRD and otitis" and "otitis only." No significant difference was observed among the 3 treatment groups. ${ }^{3}$ Heifers with clinical signs of BRD and clinical signs of otitis media.

${ }^{4}$ Heifers with clinical signs of otitis media, but no other signs of BRD.

\section{RESULTS}

A total of 1,567 heifers from 11 dairies were enrolled in the study. Breeds of heifers were Holstein, Jersey, and Holstein-Jersey cross. No significant difference $(P$ $>0.05)$ was observed among the 3 experimental groups for pre-enrollment variables (Table 4). Gamithromycin was administered to 631 heifers; tulathromycin was administered to 621 heifers; and no injection was administered to 315 heifers (Tables 4 and 5). At this calf-ranch, Jersey or Holstein-Jersey cross heifers were routinely commingled and were penned separately from Holstein heifers. One heifer in the negative control group and 2 heifers in the group assigned gamithromycin died after enrollment and within the 2-d interval designated for no treatment (Table 5). Therefore, the number of heifers that were eligible for treatment because of BRD in the negative control group was 314, in the group medi-

Table 4. Allocation and pre-enrollment variables: means (SEM) by group and $P$-values for the overall effect of experimental treatment (i.e., null hypothesis that no difference was present among groups)

\begin{tabular}{lcccc}
\hline Item $^{1}$ & Control & Tulathromycin & Gamithromycin & $P$-value \\
\hline Number enrolled & 315 & 621 & 631 & - \\
Holstein (\%) & $87.94(1.84)$ & $87.12(1.34)$ & $88.11(1.29)$ & 0.86 \\
Jersey (\%) & $5.40(1.27)$ & $4.51(0.83)$ & $4.28(0.81)$ & 0.74 \\
Cross-breed (\%) & $6.67(1.41)$ & $8.37(1.11)$ & $7.61(1.06)$ & 0.65 \\
Total serum protein (g/dL) & $6.35(0.05)$ & $6.35(0.03)$ & $6.33(0.03)$ & 0.87 \\
Birth weight (kg) & $37.66(0.305)$ & $37.54(0.218)$ & $37.84(0.214)$ & 0.61 \\
Weight at enrollment (kg) & $89.0(0.555)$ & $89.67(0.395)$ & $89.48(0.391)$ & 0.61 \\
DOF at enrollment & $80.04(0.19)$ & $80.14(0.13)$ & $80.50(0.13)$ & 0.69 \\
ADG at enrollment (kg) & $0.64(0.005)$ & $0.65(0.005)$ & $0.65(0.005)$ & 0.32 \\
Previous treatment, BRD (\%) & $41.27(2.77)$ & $39.13(1.96)$ & $40.10(1.95)$ & 0.82 \\
Previous treatment, otitis media (\%) & $37.46(2.73)$ & $34.62(1.91)$ & $33.91(1.89)$ & 0.55 \\
Previous ${ }^{2}$ treatment, diarrhea (\%) & $63.49(2.71)$ & $63.12(1.94)$ & $64.34(1.91)$ & 0.90 \\
\hline
\end{tabular}

${ }^{1} \mathrm{DOF}=$ days on feed; BRD $=$ bovine respiratory disease.

${ }^{2}$ Treated before enrollment. 
Table 5. Inventory of heifers throughout $42 \mathrm{~d}$ of observation

\begin{tabular}{|c|c|c|c|}
\hline Experimental group & Control & Tulathromycin & Gamithromycin \\
\hline Died before 1st treatment & $1^{1}$ & 0 & $2^{1,2}$ \\
\hline Eligible for 1st treatment & 314 & 621 & 629 \\
\hline Did not receive 1st treatment (success of control) & $163 / 314(51.75 \%)$ & $364 / 621(58.6 \%)$ & $411 / 629(65.13 \%)$ \\
\hline Died after 1 st treatment & $2^{2}-20$ & $1^{2}$ & $2^{2}$ \\
\hline Responded after 1st treatment & $127 / 149(84.7 \%)$ & $221 / 256(86.3 \%)$ & $189 / 216(87.5 \%)$ \\
\hline Died after 2 nd treatment & 0 & $2^{2}$ & $1^{2}$ \\
\hline Responded after 2 nd treatment & $22 / 22(100 \%)$ & $30 / 33(90.9 \%)$ & $23 / 26(88.5 \%)$ \\
\hline Eligible for 3rd treatment & 22 & 33 & 26 \\
\hline Received 3rd treatment & $0 / 22(0 \%)$ & $3 / 33(9.1 \%)$ & $3 / 26(11.5 \%)$ \\
\hline Died after 3 rd treatment & 0 & 0 & $1^{2}$ \\
\hline Responded after 3rd treatment & $\mathrm{NA}^{4}$ & NA & $1 / 2(50 \%)$ \\
\hline Received 4 th treatment & $0 / 0(0 \%)$ & $0 / 3(0 \%)$ & $1 / 2(50 \%)$ \\
\hline
\end{tabular}

${ }^{1}$ Death attributed to otitis media.

${ }^{2}$ Death attributed to pneumonia.

${ }^{3}$ Percent of heifers that received 1st treatment was calculated using the number eligible for 1st treatment. Therefore, that value is slightly different from the value recorded in Table 6 .

${ }^{4} \mathrm{NA}=$ not applicable.

cated with tulathromycin was 621 , and in the group medicated with gamithromycin was 629 . Distribution of heifers by clinical condition is presented in Tables 2 and 3. During the 42-d observation period, BRD morbidity (failure to control BRD) was less $(P<0.01)$ for heifers in the group assigned gamithromycin $(218 / 629,35 \%)$ than for heifers in the group assigned tulathromycin (257/621, 41\%; Table 6, Figure 1). Morbidity due to BRD (failure to control BRD) was also less $(P<0.01)$ for heifers that were assigned gamithromycin (218/629, $35 \%$ ) than for heifers in the negative control group (151/314, 48\%; Table 6, Figure 1). Fewer heifers ( $P$ $<0.01)$ that were medicated with either antimicrobial medication were subsequently treated because of $\mathrm{BRD}$, otitis media, or both (Table 6, Figure 2). Heifers assigned gamithromycin (mean ADG $=0.832 \pm$ SEM $0.01)$ and those assigned tulathromycin $(\mathrm{ADG}=0.841$ $\pm 0.01)$ maintained greater $(P \leq 0.01)$ ADG than did

Table 6. Outcome variables: means (SEM) by group and $P$-values for the overall effect of experimental treatment (i.e., testing the null hypothesis that no difference was present among groups) during the 42-d study

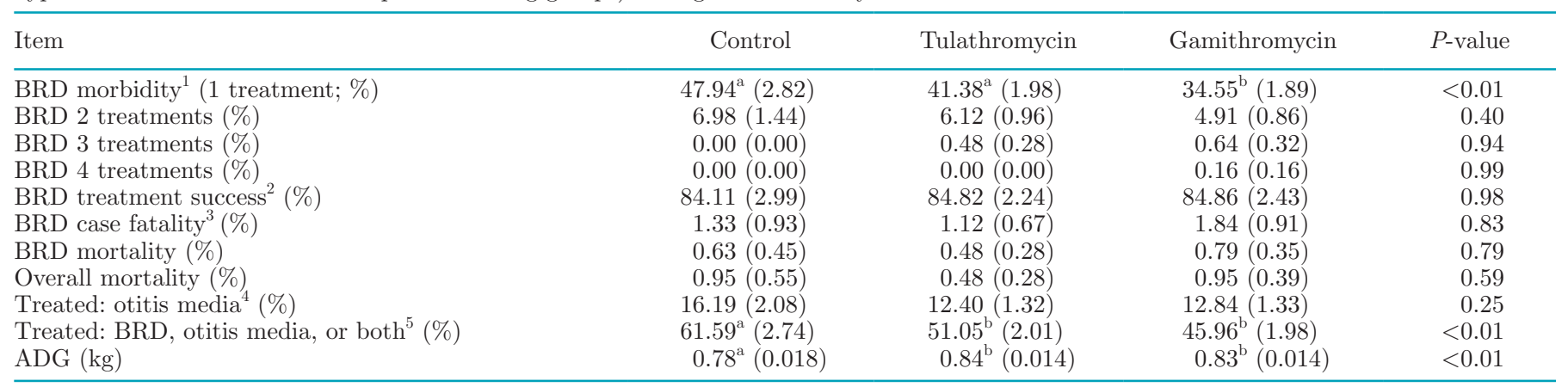

$\overline{{ }^{a}, \mathrm{~b}}$ Values with different superscripts within a row are significantly different $(P \leq 0.05)$.

${ }^{1}$ Bovine respiratory disease (BRD) with or without otitis media (BRD only + BRD and otitis; Table 2). Percent of heifers that received 1 st treatment was calculated using the number originally enrolled in the respective treatment group. Therefore, that value is slightly different from the value recorded in Table 5 .

${ }^{2}$ Treated only once because of BRD (with or without otitis media) during the study, and did not die.

${ }^{3}$ Treated at least once because of BRD (with or without otitis media) and died due to respiratory disease.

${ }^{4} \mathrm{BRD}$ and otitis + otitis only (Table 3 ).

${ }^{5}$ BRD morbidity + otitis only (Tables 2 and 3 ). 


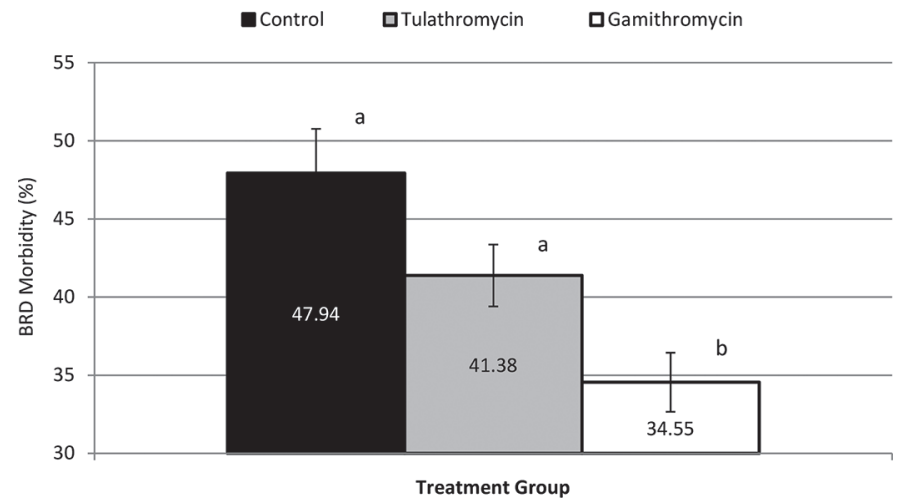

Figure 1. Mean \% ( \pm SEM) of bovine respiratory disease $(\mathrm{BRD})$ morbidity (BRD with or without otitis media) after enrollment. Values with different letters are significantly different $(P \leq 0.05)$.

heifers in the negative control group (ADG $=0.777 \pm$ 0.02; Table 6, Figure 3).

Risk assessment is a mathematical method of evaluating results of research in such a way that clinically applicable statements can be used to describe those results (Straus et al., 2005). Calculations for risk assessment are simple and apply to observational data as well as to results of hypothesis testing investigations. In this study the 2 antimicrobials reduced the risk of $\mathrm{BRD}$, with or without otitis media, in weanling dairy heifers, and gamithromycin lessened that risk to a greater degree than did tulathromycin (Table 7). During the 42-d duration of this study, the value for the number needed to treat indicated that for every 15 heifers $(95 \% \mathrm{CI}=7$ to $\infty)$ that were medicated with tulathromycin, 1 less heifer required treatment because of $\mathrm{BRD}$, with or without otitis media, than for those heifers that were not medicated. For every 7 heifers

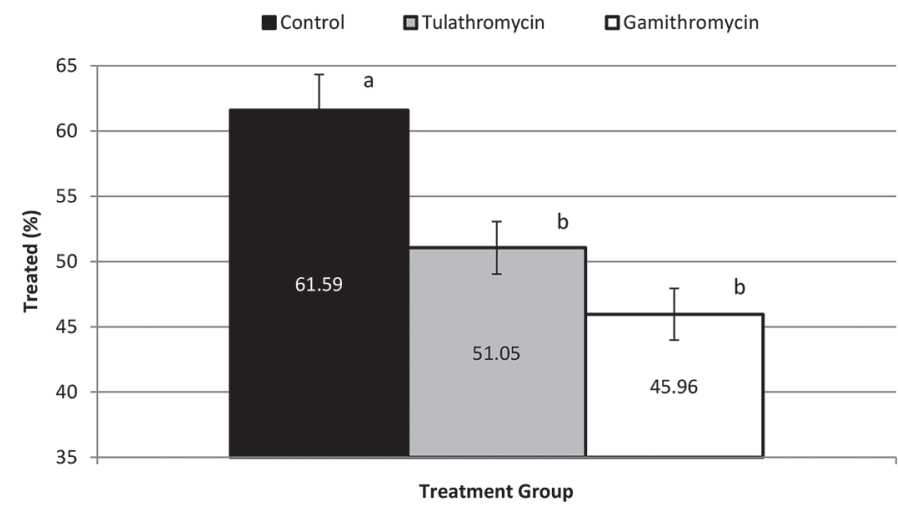

Figure 2. Mean $\%( \pm$ SEM $)$ of heifers treated because of bovine respiratory disease (BRD), otitis media, or both (BRD morbidity + otitis media) after enrollment. Values with different letters are significantly different $(P \leq 0.05)$.

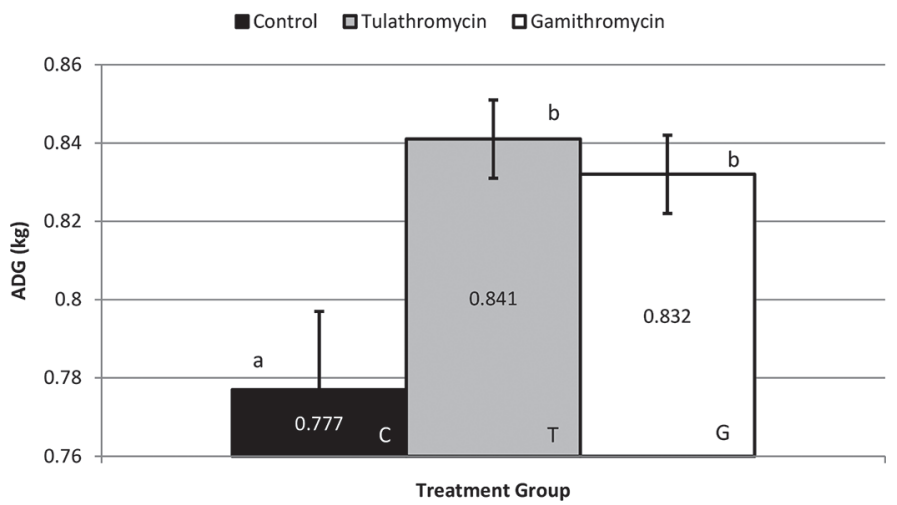

Figure 3. Mean ADG ( \pm SEM) by treatment group. Values with different letters are significantly different $(P \leq 0.05)$.

$(95 \% \mathrm{CI}=5$ to 15$)$ that received gamithromycin, 1 less heifer required treatment because of BRD, with or without otitis media, than for those that received no medication. For every 15 heifers $(95 \%$ CI $=8$ to 74) that received gamithromycin, 1 less heifer required treatment because of $\mathrm{BRD}$, with or without otitis media, than for those that received tulathromycin.

\section{DISCUSSION}

The study reported here was a clinical investigation to evaluate 2 commercially available antimicrobial products within one protocol for the control of $\mathrm{BRD}$, with or without otitis media, at one commercial facility, under climatic conditions of one 42-d time-frame. There was no intention to evaluate all possible permutations of products, protocols, climatic conditions, or years for control of BRD, otitis media, or both. Antimicrobial products used in this study are approved by the Food and Drug Administration-Center for Veterinary Medicine to control BRD in beef cattle, and in dairy cattle less than 20 mo of age that are at high risk of developing respiratory disease. Both products are also approved to treat BRD in beef cattle, and in dairy cattle less than $20 \mathrm{mo}$ of age. Labeling of approved products contains the term "control" in preference to "metaphylaxis." "Treatment" is the administration of a product or procedure after clinical signs are present; therefore, "treatment" is for sick animals, and "control" is for animals that appear clinically healthy and are at high risk of developing the disease. Some antimicrobials are approved for treatment of bacterial infections, and some are also approved for control of bacterial disease. Protocols differ for administering antimicrobials to control BRD. Comparative, controlled clinical trials have shown differences in timing of administration of the antimicrobials, duration of observation, and differences 
Table 7. Assessment of risk of failure to control bovine respiratory disease (BRD), with or without otitis media, during the 42-d observation period

\begin{tabular}{|c|c|c|c|c|c|c|}
\hline \multirow[b]{2}{*}{ Group } & \multirow[b]{2}{*}{ Success $^{1}$} & \multirow[b]{2}{*}{ Failure $^{2}$} & \multirow[b]{2}{*}{$\mathrm{RR}^{3}(\%)$} & $\operatorname{RRR}^{4}(\%)$ & $\operatorname{ARR}^{5}(\%)$ & $\mathrm{NNT}^{6}$ \\
\hline & & & & \multicolumn{3}{|c|}{$[95 \% \mathrm{CI}]$} \\
\hline Control & $163 / 314$ & 151/314 (CER) & & [14.1 to 41.8$]$ & [6.8 to 20.1$]$ & [5 to 15$]$ \\
\hline Tulathromycin & $364 / 621$ & 257/621 (EER) & 86 & 13.9 & 6.7 & 15 \\
\hline Control & $163 / 314$ & $151 / 314$ (CER) & & {$[-0.09$ to 27.98$]$} & {$[-0.04$ to 13.45$]$} & {$[7$ to $\infty]$} \\
\hline Gamithromycin & $411 / 629$ & 218/629 (EER) & 83.75 & 16.25 & 6.7 & 15 \\
\hline
\end{tabular}

${ }^{1}$ Heifers in the indicated group that did not receive treatment because of BRD with or without otitis media.

${ }^{2}$ Heifers in the indicated group that received one or more treatments because of BRD (with or without otitis media). EER = experimental event rate-incidence of failure: fraction of heifers in the principal group that received treatment because of BRD with or without otitis media. CER $=$ control event rate-incidence of failure: fraction of heifers in the comparative, or baseline, group that received treatment because of BRD with or without otitis media.

${ }^{3}$ Relative risk $(\mathrm{RR})=\mathrm{EER} / \mathrm{CER}$.

${ }^{4}$ Relative risk reduction $(\mathrm{RRR})=|\mathrm{EER}-\mathrm{CER}| / \mathrm{CER}$.

${ }^{5}$ Actual risk reduction $(\mathrm{ARR})=|\mathrm{EER} / \mathrm{CER}|$.

${ }^{6}$ Number need to treat $(\mathrm{NNT})=1 / \mathrm{ARR}$.

among products (Young, 1995; Sgoifo Rossi et al., 2010; Stanton et al., 2010; Urban-Chmiel and Grooms, 2012; Heins et al., 2014; Ives and Richeson, 2015; Hill et al., 2017; Teixeira et al., 2017). Evaluation of all permutations of those protocols was beyond the scope of the study reported here. In the United States, no products are labeled for control or treatment of otitis media in cattle.

Adequate randomization for this study was achieved as evidenced by the desired distribution of animals in experimental groups and that no significant difference $(P>0.05)$ was observed among the 3 experimental groups for pre-enrollment variables (Table 4).

For the study reported here, personnel who administered the medication at enrollment were not blinded to the individual experimental treatment because labels and protective covers for the commercial products remained in place. Separate, multi-dose, manually filled syringes were used to administer the respective products. The volume of the appropriate dose of the respective product was visually discernable and could not be obscured. The same individuals observed and treated (according to written protocols prescribed) the heifers enrolled in this study, as well as other animals at the facility that were not enrolled in this study. Fidelity of information important to blinding was reliant on the expectation that the personnel would not remember which individual animal received what medication. Blinding of the elements mentioned above was discussed before the study began; however, logistics to do so were beyond available resources at the time.

For the products used in the study reported here, data in Freedom of Information files were for administration of the respective product after arrival of transported beef cattle (Freedom of Information Summary, 2005, 2011). Data in those files for the products illustrate the respective efficacy to control BRD in beef cattle at high risk of developing BRD. With an imposed interval of $14 \mathrm{~d}$ postadministration of tulathromycin (Freedom of Information Summary, 2005), approximately $13 \%$ (53/399 head) developed BRD versus 59\% (236/402 head) that received saline as a negative control. The proportion of animals that developed BRD in 2 studies, each with an imposed interval of $10 \mathrm{~d}$ postadministration of gamithromycin (Freedom of Information Summary, 2011), was, respectively, 15\% (15/106 head) versus $64 \%(34 / 53$ head) that received saline, and $22 \%$ $(34 / 154$ head) versus $42 \%$ (64/154 head) that received saline.

In the study reported here, the percentage of heifers that were treated because of BRD morbidity was greater than those estimated for calculation regarding design and number of animals to enroll, and those of the studies reported in the respective Freedom of Information file. One reason for that may be that dairy heifers in this study were restricted to a 2-d interval before they were eligible to be treated. This presents an opportunity to train personnel about overly aggressive treatment of animals that arguably might not have needed treatment.

A discussion of the pathogenesis of BRD is beyond the scope of this article. Bovine respiratory disease is a complex, infectious clinical condition that has been associated with multiple bacteria (Mannheimia haemolytica, Pasteurella multocida, Histophilus somni, and Mycoplasma bovis), viruses (bovine viral diarrhea virus types I and II, bovine herpesvirus-1, para-influenza 3 virus, and bovine respiratory syncytial virus), stressors, 
and other interactive factors that influence the course of BRD (Rice et al., 2007; Fulton, 2009; Taylor et al., 2010). Antimicrobial medications are part of the management tools and are only expected to act against the bacterial pathogens associated with BRD because they have no activity against viruses. Each management tool should be viewed as unique and not considered as a replacement for other tools.

This study was not powered to separately analyze effects of experimental treatments on otitis media. However, results show that co-morbidity because of otitis media was, at least partially, lessened by the antimicrobial medications administered for control of BRD, with or without otitis media. That is in contrast to findings of Stanton et al. (2013) with pre-weaning heifers. Otitis media deserves specific attention for development of products and management practices that prevent, control, and treat upper respiratory infections of cattle. Until products receive appropriate approvals, treatment of otitis media in calves constitutes extra-label use of antimicrobials and should follow the respective guidelines (Food and Drug Administration, 1996, 21 CFR Part 530). The requirement for a veterinarian-client-patient relationship must be recognized. Principal bacterial pathogens involved with otitis media of calves, and of yearlings, are found in the upper respiratory tract of healthy cattle and are associated with BRD as well. Among them are Histophilus somni, Pasteurella multocida, Mannheimia haemolytica, Trueperella pyogenes, Mycoplasma bovis, and other Mycoplasma spp., Streptococcus spp., and Actinomyces spp. (McEwen and Hulland, 1985; Yeruham et al., 1999; Duarte and Hamdan, 2004; Francoz et al., 2004; Stanton et al., 2013). Otitis media may exacerbate the severity of infections of the respiratory tract and may enhance mortality attributed to pneumonia. The pathogenesis of infections of the middle ear in calves is not fully understood. The most common means of entry of organisms into the middle ear from the pharynx is via the Eustachian tube. Infections of the middle ear may progress to internal otitis, and rupture the tympanic membrane with discharge into the external ear. Perineural dissemination of the infection can lead to development of facial paralysis, vestibulocochlear neuropathy, meningitis, or a combination of these. Osseous necrosis may occur as a consequence of chronic osteomyelitis.

Abnormal behaviors of sick animals include lethargy, inappetence, and decreased consumption of water (Preston, 2007; Hanzlicek et al., 2010; White et al., 2012; Theurer et al., 2013; Noffsinger et al., 2015). Those abnormal behaviors can cause measurable dehydration (shrink), poor weight gain, or both; however, those behaviors do not correlate with the severity of pulmonary lesions (Hanzlicek et al., 2010; Capik et al., 2017). Preston (2007), Hanzlicek et al. (2010), White et al. (2012), Theurer et al. (2013), Noffsinger et al. (2015), and Capik et al. (2017) have stated that ADG was a reflection of eating behavior. In short, animals that maintained healthy eating behavior had greater ADG. In the study reported here, the ADG during the observation period was calculated for individual animals that survived, summarized for the experimental groups, and statistically analyzed. Feed consumption, feed efficiency, feeding activity, or metabolic activity was not measured for the study reported here.

A "steward" is defined as "a person entrusted with the management of the affairs of others," and "animal husbandry" is the care and management of animals (The New International Webster's Dictionary Thesaurus and Atlas, 2006). Implicit to stewardship of animal resources are behaviors that optimize the caretaker's knowledge and abilities for the well-being of animals. Also implicit is the obligation of the caretaker to increase knowledge and understanding of practices, products, or technologies (or a combination of these) that can contribute to the well-being of animals entrusted to their care. Therefore, caretakers are obligated to take action as their knowledge/understanding changes. The health and well-being of animals can be protected with management procedures that include the appropriate use of biological and antimicrobial products to prevent, control, and treat indicated infectious diseases (Gorden and Plummer, 2010).

Stewardship of the products used to maintain the health and well-being of animals requires continued efforts to understand the multiple factors that influence how best to apply them (American Association of Bovine Practitioners, 2013, 2017; Weese et al., 2015; American Veterinary Medical Association, 2018). Included factors are mechanisms of action of those products, management changes that may need to be implemented to use those products optimally, and ongoing nonbiased evaluation of those factors.

Written protocols for use of antimicrobials approved to control BRD should be customized by attending veterinarian(s) as needed for specific facilities (calf ranches; Gorden and Plummer, 2010). Personnel at those facilities should be trained to monitor animals, review records, and trained to follow protocols for control or treatment (or both) of BRD when criteria are met. Communication among veterinarians, management personnel, and caretakers should be as frequent as appropriate for the need. Those criteria are often developed based on historical data specific to that facility, published scientific data, results of studies performed at that facility, or a combination of these. 


\section{CONCLUSIONS}

Under the conditions of this study, the use of antimicrobial medication to control BRD, with or without otitis media, in weaned dairy heifers had health and ADG benefits. Gamithromycin was superior to tulathromycin for control of BRD and BRD with co-morbidity by otitis media. Either antimicrobial was superior to no medication for subsequent treatment because of BRD, otitis media, or both. Mortality was not different among the treatment groups. Stewardship of animal care and animal health products requires that protocols for control of BRD, with or without otitis media, be evaluated and improved to achieve optimal results and to judiciously use antimicrobials as a management tool. This research, because it provides new information on the relative benefit of different treatments for control of BRD in dairy calves, provides information that producers can use in support of making well-informed decisions that promote good stewardship. Metaphylaxis with antimicrobial medication is a valid and approved method for control of $\mathrm{BRD}$, with or without otitis media, in cattle at high risk of developing BRD. As with use of any medication, evaluation of the protocols (product, dose, route of administration, site of administration, dosing frequency, pre-slaughter withdrawal time) and of the efficacy should be an important part of refinement of management procedures to protect and maintain the health and well-being of dairy heifers.

\section{ACKNOWLEDGMENTS}

This clinical investigation was supported by Merial, now part of Boehringer-Ingelheim Animal Health (Duluth, GA). Gordon W. Brumbaugh performed services as per contract with Merial, now part of BoehringerIngelheim Animal Health.

\section{REFERENCES}

American Association of Bovine Practitioners. 2017. Key elements for implementing antimicrobial stewardship plans in bovine veterinary practices working with beef and dairy operations. Accessed Apr. 19, 2018. http://aabp.org/resources/AABP_Guidelines/ AntimicrobialStewardship-7.27.17.pdf.

American Association of Bovine Practitioners. 2013. Prudent antimicrobial use guidelines for cattle. Accessed Apr. 19, 2018. http://aabp.org/resources/aabp_guidelines/AABP_Prudent _Antimicrobial_Use_Guidelines-2013.pdf.

American Veterinary Medical Association. 2018. Antimicrobial stewardship definition and core principles. Accessed Apr. 19, 2018. http://www.avma.org/News/JAVMANews/Pages/180301d.aspx.

Bach, A. 2011. Associations between several aspects of heifer development and dairy cow survivability to second lactation. J. Dairy Sci. 94:1052-1057. https://doi.org/10.3168/jds.2010-3633.

Beef Quality Assurance. 2013. Dairy animal care and quality assurance. Accessed Apr. 19, 2018. https://www.bqa.org/Media/BQA/ Docs/dairybqamanual.pdf.
Capik, S. F., B. J. White, R. L. Larson, N. Van Engen, and J. F. Coetzee. 2017. Effect of meloxicam administration on movement, feeding, and drinking behaviors of transported and nontransported cattle. Am. J. Vet. Res. 78:1437-1443.

Duarte, E. R., and J. S. Hamdan. 2004. Otitis in cattle, an aetiological review. J. Vet. Med. B Infect. Dis. Vet. Public Health 51:1-7.

Food and Drug Administration. 1996. Department of Health and Human Services. Extra-Label Drug Use in Animals; Definitions. Accessed Apr. 19, 2018. 21 CFR Part 530; Subpart A; 530.3 - Definitions. https://www.govinfo.gov/content/pkg/FR-1996-11-07/pdf/ 96-28662.pdf.

Francoz, D., G. Fecteau, A. Desrochers, and M. Fortin. 2004. Otitis media in dairy calves: A retrospective study of 15 cases (1987 to 2002). Can. Vet. J. 45:661-666.

Freedom of Information Summary. 2005. NADA 141-244 Draxxin Injectable Solution - Original New Animal Drug Application. Accessed Mar. 4, 2019. https://animaldrugsatfda.fda.gov/adafda/ app/search/public/document/downloadFoi/789.

Freedom of Information Summary. 2011. NADA 141-328 Zactran Gamithromycin Injectable Solution - Original New Animal Drug Application. Accessed Mar. 4, 2019. https://animaldrugsatfda.fda .gov/adafda/app/search/public/document/downloadFoi/886.

Fulton, R. W. 2009. Bovine respiratory disease research (1983-2009). Anim. Health Res. Rev. 10:131-139.

Gorden, P. J., and P. Plummer. 2010. Control, management, and prevention of bovine respiratory disease in dairy calves and cows. Vet. Clin. North Am. Food Anim. Pract. 26:243-259. https://doi.org/ 10.1016/j.cvfa.2010.03.004.

Guterbock, W. M. 2014. The impact of BRD: The current dairy experience. Anim. Health Res. Rev. 15:130-134. https://doi.org/10 $.1017 /$ S1466252314000140.

Hanzlicek, G. A., B. J. White, D. Mosier, D. G. Renter, and D. E. Anderson. 2010. Serial evaluation of physiologic, pathological, and behavioral changes related to disease progression of experimentally induced Mannheimia haemolytica pneumonia in postweaned calves. Am. J. Vet. Res. 71:359-369.

Heins, B. D., D. V. Nydam, A. R. Woolums, R. D. Berghaus, and M. W. Overton. 2014. Comparative efficacy of enrofloxacin and tulathromycin for treatment of preweaning respiratory disease in dairy heifers. J. Dairy Sci. 97:372-382. https://doi.org/10.3168/ jds.2013-6696.

Hill, T. M., J. D. Quigley, F. X. Suarez-Mena, T. S. Dennis, and R. L. Schlotterbeck. 2017. Case study: Control of bovine respiratory disease in dairy calves with tulathromycin and effect on calf health and performance from 0 to 4 months of age. Prof. Anim. Sci. 33:498-503. https://doi.org/10.15232/pas2017-01625.

Ives, S. E., and J. T. Richeson. 2015. Use of antimicrobial metaphylaxis for the control of bovine respiratory disease in high-risk cattle. Vet. Clin. North Am. Food Anim. Pract. 31:341-350. https://doi .org/10.1016/j.cvfa.2015.05.008.

McEwen, S. A., and T. J. Hulland. 1985. Haemophilus somnus-induced otitis and meningitis in a heifer. Can. Vet. J. 26:7-8.

Noffsinger, T., K. Lukasiewicz, and L. Hyder. 2015. Feedlot processing and arrival cattle management. Vet. Clin. North Am. Food Anim. Pract. 31:323-340. https://doi.org/10.1016/j.cvfa.2015.06.002.

Preston, R. L. 2007. Receiving cattle nutrition. Vet. Clin. North Am. Food Anim. Pract. 23:193-205.

Rice, J. A., L. Carrasco-Medina, D. C. Hodgins, and P. E. Shewen. 2007. Mannheimia haemolytica and bovine respiratory disease. Anim. Health Res. Rev. 8:117-128. https://doi.org/10.1017/ S1466252307001375.

Schaffer, A. P., R. L. Larson, N. Cernicchiaro, G. A. Hanzlicek, S. J. Bartle, and D. U. Thomson. 2016. The association between calfhood bovine respiratory disease complex and subsequent departure from the herd, milk production, and reproduction in dairy cattle. J. Am. Vet. Med. Assoc. 248:1157-1164.

Sgoifo Rossi, C. A., S. L. Vandoni, M. Bonfanti, and A. B. Forbes. 2010. Effects of arrival medication with gamithromycin on bovine respiratory disease in feedlot cattle in Italy. Int. J. Appl. Res. Vet. Med. 8:87-96. 
Stanton, A. L., D. F. Kelton, S. J. Leblanc, S. T. Millman, J. Wormuth, R. T. Dingwell, and K. E. Leslie. 2010. The effect of treatment with long-acting antibiotic at postweaning movement on respiratory disease and on growth in commercial dairy calves. J. Dairy Sci. 93:574-581. https://doi.org/10.3168/jds.2009-2414.

Stanton, A. L., D. F. Kelton, S. J. LeBlanc, J. Wormuth, L. K. Fox, and K. E. Leslie. 2013. Effects of tulathromycin on incidence of various diseases and growth of young heifers. J. Am. Vet. Med. Assoc. 243:267-276. https://doi.org/10.2460/javma.243.2.267.

Stanton, A. L., D. F. Kelton, S. J. LeBlanc, J. Wormuth, and K. E. Leslie. 2012. The effect of respiratory disease and a preventative antibiotic treatment on growth, survival, age at first calving, and milk production of dairy heifers. J. Dairy Sci. 95:4950-4960. https: //doi.org/10.3168/jds.2011-5067.

Straus, S. E., W. S. Richardson, P. Glasziou, and R. B. Haynes. 2005. Evidence-Based Medicine - How to Practice and Teach EBM. 3rd ed. Elsevier Churchill Livingstone, Philadelphia, PA

Taylor, J. D., R. W. Fulton, T. W. Lehenbauer, D. L. Step, and A. W. Confer. 2010. The epidemiology of bovine respiratory disease: What is the evidence for predisposing factors? Can. Vet. J. 51:1095-1102.

Teixeira, A. G. V., J. A. A. McArt, and R. C. Bicalho. 2017. Efficacy of tildipirosin metaphylaxis for the prevention of respiratory disease, otitis and mortality in pre-weaned Holstein calves. Vet. J. 219:44-48. https://doi.org/10.1016/j.tvjl.2016.12.004.

The New International Webster's Dictionary Thesaurus and Atlas. 2006 ed. S. I. Landau, ed. Trident Reference Publishing, Naples, FL.

Theurer, M. E., D. E. Amrine, and B. J. White. 2013. Remote noninvasive assessment of pain and health status in cattle. Vet. Clin. North Am. Food Anim. Pract. 29:59-74.

University of California-Davis. 2016. Bovine respiratory disease scoring system for pre-weaned dairy calves. Accessed Apr. 19, 2018 https://www2.vetmed.ucdavis.edu/vmtrc/local_resources/pdfs / BRD_ANR_Brochure_Nov\%202016\%20FINAL.PDF.
Urban-Chmiel, R., and D. L. Grooms. 2012. Prevention and control of bovine respiratory disease. J. Livest. Sci. 3:27-36.

USDA-APHIS-VS-CEAH-NAHMS. 2012. 2011 Dairy Heifer Raiser. Animal Health Research Reviews 613.1012. Accessed Mar. 4. 2019. http://www.aphis.usda.gov/animal_health/nahms/dairy/ downloads/dairyheifer11/HeiferRaiser.pdf.

Walker, W. L., W. B. Epperson, T. E. Wittum, L. K. Lord, P. J. Rajala-Schultz, and J. Lakritz. 2012. Characteristics of dairy calf ranches: Morbidity, mortality, antibiotic use practices, and biosecurity and biocontainment practices. J. Dairy Sci. 95:2204-2214. https://doi.org/10.3168/jds.2011-4727.

Weese, J. S., S. Giguère, L. Guardabassi, P. S. Morley, M. Papich, D. R. Ricciuto, and J. E. Sykes. 2015. ACVIM consensus statement on therapeutic antimicrobial use in animals and antimicrobial resistance. J. Vet. Intern. Med. 29:487-498.

Wells, S. J., L. P. Garber, and G. W. Hill. 1997. Health status of preweaned dairy heifers in the United States. Prev. Vet. Med. 29:185-199.

White, B. J., D. E. Anderson, D. G. Renter, R. L. Larson, D. A. Mosier, L. L. Kelly, M. E. Theurer, B. D. Robert, and M. L. Walz. 2012 Clinical, behavioral, and pulmonary changes in calves following inoculation with Mycoplasma bovis. Am. J. Vet. Res. 73:490-497.

Windeyer, M. C., K. E. Leslie, S. M. Godden, D. C. Hodgins, K. D Lissemore, and S. J. BeBlanc. 2014. Factors associated with morbidity, mortality, and growth of dairy heifer calves up to 3 months of age. Prev. Vet. Med. 113:231-240. https://doi.org/10.1016/j .prevetmed.2013.10.019.

Yeruham, I., D. Elad, and M. Liberbiom. 1999. Clinical and microbiological study of an otitis media outbreak in calves in a dairy herd. J. Vet. Med. B Infect. Dis. Vet. Public Health 46:145-150.

Young, C. 1995. Antimicrobial metaphylaxis for undifferentiated bovine respiratory disease. Comp. Cont. Educ Pract. Vet. 17:133142. 